Monatsschrift f. Geburtshülfe u. Gynäkologie 1915;41:454-456

\title{
Literatur- Verzeichnis
}

Geburtshülfe. Küstner, Rob. von Olshausen †. Zbl. f. Gyn. 20. III. S. 177.

Bloägett, S. H., Zur Prophylaxe puerperaler Krämpfe. Med. Rec. 20. III.

S. 478.

Litera tur- Verzeichnis.

455

Corletto, F., Ein neuer Fall von Osteomalacie, geheilt nach Bossi. Gin. mod.

Aug.-Dez. 1914. S. 286. Ebeler und Löhnberg, Weitere Eríahrungen mit der Abderhaldensch $\beta n$

Fer-

mentreaktion. Berl. klin. Woch. 29. III. S. 319. Eggstein, A. A., Die Abderhaldensche Reaktion. Journ. of Amer. med.

Assoc. 27. II. S. 735. Fantuerí, Offener Brief an Prof. Bossi über die Heilung der Osteomalacie naeh der Met·hode Bossi. Gin. mod. Ang.-Dez. 1914. S. 283. Hohlweg, H., Der Reststickstoff des Bhites unter physiologischen Bedin-

gtingen, sein Verhalten bei Nephritis, Ilrämie und Eklampsie, sowie

seine Bedeutung für die Prüfung der Nierenfunktion. Med. Klinik.

21. III. S. 331. Lange, Joh., Isochronisch lieterotope Eiimplantation bei Myoma uteri und dadurch bedingter Retroderiation des Gebärorgans. Ztbl. f. Gyn.

27. III. S. 201. Liesegang, lî. E., Über die puerperale Osteomalacie. Zbl. f. Gyn. 10. IV.

S. 241. Mayer, Paul, Die Eklampsiefälle der Heidelberger Frauenklinik in den

Jahren 1902 -1912. Med. Blatter. Nr. 5 u. 6. 5. III.-20. III. Menton, W. P., Die Friihdiagnose der Verletzvmgen des Gebärschlauches.

Arch, of Diagnosis. New York. Okt. 1914. Rißmann, P., VVeitere Beiträge zur diätetischen und medikamentösen

Beeinflassung dér Schwangerschaft und Eklampsiebehandlung. Med.

K1. 11. IV. S. 427. Wallace, C. J'., Ruptura appendicis am normalen Sehwangerschaftsende.

Journ. of Amer. med. Assoc. 27. II. S. 739. Zalewski, Über Placenta praevia v́nd die Vorteile der ävißeren $\lambda$ Vendung

dabei. Arch, f. Gyn. Bd. 104. S. 133. Zangemeister und Kirstein, Zur Frage der Selbstinfektion. Arch. f. Gyn.

Bd. 104. > S. 1.

Neugebornes.

Mayer, A., Über den Zusammenhang zwischen Gíewichtsabnahme und Temperatursteigerungen beim Neugeborenen (Hungorfieber). Arcli. f. Gyn. Bd. 101. S. 159.

Spiller, W. G., Icterus gravis neonati als tTrsache cerebraler $\mathrm{Kr}^{1} / 8 \mathrm{mpfe}$. Amer. Journ. of the med. Sc. März 1915. S. 345.

Wínkler, F., und von ßehr-Pinnow, Statistische Beiträge für die Beur-teilung der Säuglingssterblichkeit in Preußen. Unter Benutzung von amtlichem Material. Verlag des Kaiserin-Auguste-Victoria-Hauses zur Bekämpfung der Säuglingssterblichkeit im Deutschen Reich in Chariottenburg. 1915. 
Gynäkologíe.

Andeus, Über die Wirkung einiger Uterustonika auf die Zirkulation mit spezieller Berücksichtigung des kleinen Kreislaufes. Arch. f. Gyn.

Bd. 104. S. 103. ..-iMÒerí-Gent, Fibrom des Eierstocks mit cystischer Entartung. Frauenarzt. H. 3. Jahrg. XXX. S. 76. Bab, H., Zur medikamentösen Behandlung der innersekretorischen $\mathrm{Ov}^{3} / 4$ rialinsuffizienz. Med. K1. 11. IV. S. 429.

456

Lit eratur- Verzeichnis.

Block, J., Erwiderung auf vorstehende Bemerkungen. Med. Kl. 11. IV.

S. 430. Baldassari, Heilung gynäkologischer Leiden bei Geisteskrankheiten. Lucina. 1. IV. S. 49. Campione, F., Uterusfibrom, kompliziert durch entzündhche Adnex-Erkrankungen. Gin. mod. Aug.-Dez. 1914. S. 290. Columbine-, C, München, Über Transplantation der Ovarien beim Menschen.

Gyn. Rundsch. 1914. H. 23. Grüner, Ed., Die Beziehungen der Menstruation, der

Schwangerschaft, der Geburt, des Wochenbetts und des Klimakterium zu Erkran-

kungen der Zähne. Dtsch. Zahnheilk. H. 34. S. 9. Jaschke, B. Th., Zur Diagnose und Therapie der Xierentuberkulose bei

Frauen. Ztschr. f. gyn. Urol. Bd. V. H. 1. Kalliski, D. J., Kanüle und Nad el für BKittransfusion und intravenose

Infusion. Med. Rec. 20. III. S. 482. Kubinyi, P. v., Budapest, Zur Cystokopie der Blasenfisteln. Ztschr. f.

gyn. Urol. Bd. V. H. 1. Leví, G., Bemerkungen über eine Fissura labio-maxillo-palatina bei einem

menschlichen Embryo. Gin. mod. Atig.-Dez. 1914. S. 260. Moscateïli, C, Urteil eines praktischen Arztes über den Dilatator Bossi.

Gin. mod. Aug.-Dez. 1914. S. 258. Ottow, B-, Dorpat, Zur Embryologie der

Ureterenverdoppelung und die

Bedeutung der letzteren für die Pathologie der Nieren. Ztschr. f.

gyn. Urol. Bd. V. H. 1. Romeo, P., Die verschiedenen Methoden der Cervixerweiterung und der Dilatator Bossi. Gin. mod. Aug.-Dez. 1914. S. 253. Rueck, G. A., Bluttransfusion nach der Gravitationsmethode. Med. Rec.

27. II. S. 354. Schroder, Anatomische Studien zur normalen und pathologischen Physiologie des Menstruationszyklus. Arch. f. Gyn. Bd. 104. S. 27. Spitzig, B. L., Zitronensaure Salze bei kongestiver Dysmenorrhoe: die

Beziehung dieser zu dem Status vagotonicus. The Journ. of Amer,

med. Assoc. 27. II. S. 733. Stoeckel, Die extraperitoneale Tubenverlagerung als Methode der Sterili-

sierung. Zbl. f. Gyn. Bd. III. S. 161. Walther, Thigasin „Henning”. Eine anaesthesierende Thigenolsalbe.

Frauenarzt. Jahrg. XXX. H. 3. S. 66. Zeckete, A., Über den Wert der Ä > ssí'-Operation bei Dysmenorrhoe und

Sterilität. Gin. mod. Aug.-Dez. 1914. S. 277.

Strahlentherapie.

Albers-Schönberg und Lorenz, Die Schutzmittel für Arzte und Personal 
bei der Arbeit mit Röntgenstrahlen. Dtsch. med. Woch. 11. III.

S. 301. Döderlein, Zur Strahlenbehandlung des Krebses. Zbl. f. Gyn. Nr. 12. Derselbe,

Strahlentherapie bei Karzinom. Beitr. z. kl. Chir. XCV. H. 3. Füth H., und Ebeler, T., Röntgenund Radiumtherapie des Uterus-

karzinom. Zbl. f. Gyn. 3. IV. S. 217. Salzmann, Sekundärstrahlen in der Röntgentiefentherapie als Ersatz

r.Substanzen. radioaktiver Substanzen. Dtsch. med. Woch. 18. II. S. 223.

Verantwortlicher Redakteur: A. Martin in Berlin. 